\title{
El bovino Criollo Argentino: principales características de la raza
}

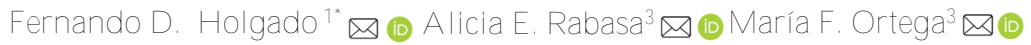 \\ Facultad de Agronomía y Zootecnia. Universidad Nacional de Tucumán, Argentina.
}

\section{The A rgentine Criollo cattle: main characteristics of the breed}

\begin{abstract}
A bstract. The Argentine Creole cattle have its origins in the cattle introduced to America by the European during colonization. Phenotypically it is characterized by the great diversity of coat colors and its horns, although there are also animals without it. The breed is also characterized by its relatively small body size and moderate milk aptitude, so that the breeding cows have low energy requirements for maintenance and production. In adverse nutritional environments, the Argentine Creole has a high response capacity. It is distinguished for its fertility, calving ease, breeding capacity, longevity and resistance to different environmental factors, considering itself as a breed specialized in calves' production. Under the same production conditions, the butcher performance of the beef and the quality of meat produced by Creole animals is similar to that of other bovine breeds. This animal genetic resource is considered of great value, and must be preserved due to its genetic variability to face current and future production challenges. The use of genetic resources is the best way to maintain the animals' diversity, and Argentine Creole race have excellent qualities to improve cattle breeding in specific environments or through crossbreeding. It is crucial to consider the definition of strategies that favor their diffusion and use at the country level.
\end{abstract}

Key words: A nimal genetic resources, Livestock, Genetic diversity.

Resumen. El bovino Criollo Argentino tiene sus orígenes en el ganado introducido a América por los españoles durante la colonización. Fenotípicamente se caracteriza por la gran diversidad de colores de capa y por su cornamenta, aunque existan además animales sin cuernos. La raza se caracteriza también por su tamaño corporal relativamente chico y una aptitud lechera moderada, por lo que los vientres de cría poseen bajos requerimientos energéticos de mantenimiento y producción. En ambientes nutricionales adversos, el Criollo Argentino posee alta capacidad de respuesta. Se destaca por su fertilidad, facilidad de parto, capacidad de cría, longevidad y resistencia a diferentes factores ambientales, considerándose como una raza especializada en la producción de terneros. Bajo iguales condiciones de producción, el rendimiento carnicero de la res y la calidad de carne producida por animales Criollos es similar al de otras razas bovinas. Este recurso zoogenético es considerado de gran valor, debiendo ser preservado por su variabilidad genética para hacer frente a desafíos productivos actuales y futuros. La utilización de los recursos genéticos es el mejor camino para el mantenimiento de la diversidad de los animales domésticos, y el bovino Criollo Argentino posee excelentes cualidades para mejorar la ganadería vacuna en determinados ambientes o a través de cruzamientos. A nivel país, es importante considerar la definición de estrategias que favorezcan su difusión y utilización.

Palabras claves: Recursos zoogenéticos, Ganadería, Diversidad genética.

\section{Introducción}

\section{El bovino Criollo A rgentino}

La primera introducción de bovinos al continente Americano se produce en el segundo viaje de Colón a la isla La Española, hoy República Dominicana, en el año 1493. Este ganado provenía del Sudeste de la Península I bérica y de las Islas Canarias (Wilkins et al., 1982). Cincuenta y seis años después de su arribo a América, los vacunos llegan al Virreinato del Río de la Plata (Argentina); de manera que durante tres siglos, que se extienden desde el año 1549 a mediados del siglo XIX, en nuestras tierras había sólo ganado bovino Criollo.

En este sentido, reconocemos por Criollo a todos aquellos animales descendientes del ganado introducido por los españoles que pueblan diferentes países del continente americano, desde los EEUU hasta la Patagonia A rgentina.

Recibido: 2020-07-27. A ceptado: 2021-03-10.

${ }^{1}$ A utor para la correspondencia fernandodholgado@gmail.com

${ }^{2}$ A sociación Argentina de Criadores de Ganado Bovino Criollo (AACGBC).

${ }^{3}$ Instituto de Investigación A nimal del Chaco Semiárido (CIAP - INTA Leales) Tucumán, Argentina. 
A partir del año 1850 en la Pampa Húmeda Argentina se inicia la introducción de razas bovinas británicas, en un inicio Shorthorn y luego Hereford y A berdeen Angus, con el objetivo de obtener un animal con mayor cantidad de grasa y preferible conformación en un sentido convencional. Los cruzamientos generados con el ganado autóctono fueron sumamente exitosos, interpretándose en ese entonces, que se debía a la calidad de las razas introducidas y no al concepto de vigor híbrido de estas primeras cruzas (Filial 1). De esta manera, el avance de las razas británicas determinó la absorción total del Criollo en la Pampa Húmeda Argentina y su desplazamiento hacia zonas marginales, especial mente el Noroeste; donde el calor, las garrapatas y el monte obstaculizaron el desarrollo de las razas introducidas hasta el momento. Con la introducción de razas cebuinas un siglo después, es que la presencia del bovino Criollo se ve realmente amenazada a nivel nacional, con la posibilidad de ser absorbido progresivamente por el ganado exótico.

En el año 1956 en Argentina se crea el Instituto Nacional de Tecnología Agropecuaria (INTA) con el objetivo de investigar y difundir nuevas tecnologías para el productor agropecuario (https:/ / inta.gob.ar/ paginas/ historia-del-inta). La Estación Experimental de Leales, ubicada en la provincia de Tucumán comienza sus actividades en el año 1959, con la finalidad de evaluar cruzamientos con ganado Cebú (Nelore) y su comparación con los bovinos existentes en la región denominados Criollos. Se conforma de esta manera el primer plantel experimental de animales Criollos compuesto de 35 vacas y 2 toros, que fueron adquiridos en la provincia de Salta, y que luego se enriqueció con nuevas introducciones desde las provincias de Jujuy, Chaco, Santiago del Estero y Tucumán.

En búsqueda de definir al ganado Criollo, en el año 1970 se realiza una encuesta a productores y técnicos que se consideraban expertos calificados en la temática, llegando a la conclusión de que Criollo era todo aquel ganado mestizo que no se parecía a ninguna raza conocida. Además, se planteó la necesidad de caracterizar este material genético en base a cuatro aspectos: morfológico, productivo, genético y etológico; lo que dio origen a la publicación de cinco libros y una importante cantidad de trabajos científicos por parte de INTA y de distintas Universidades de nuestro país.

En el año 1984, el jefe de la Agencia de Extensión Rural de INTA Jesús María (provincia de Córdoba), Ing. Agr. Gonzalo Ruiz Sempere y un productor de la zona, Dr. Enrique Andreani, visitan el plantel de bovinos Criollos de INTA Leales con el objetivo de conocer la raza (Carrazzoni, 1998). Esta visita dio lugar a la Primera Jornada de ganado bovino Criollo, llevada a cabo en Córdoba ese mismo año, y en la que se impulsó la formación de la A sociación de Criadores de Bovinos Criollos un año después. Al día de hoy la A sociación nuclea a los productores de distintas zonas del país, difunde las características de la raza a través de jornadas, exposiciones, etc., y es responsable de los registros geneal ógicos denominados Base.

El Criollo Argentino ha sido aceptado como Raza por la Sociedad Rural Argentina en el año 1990, y desde entonces participa de numerosas exposiciones ganaderas, entre ellas la Exposición Internacional de Pal ermo, vidriera productiva más importante del país.

En la actualidad, el bovino Criollo Argentino habita todo el territorio del país, desde la región de la Puna hasta la Patagonia constituyendo una población única con características comunes. Debido al aislamiento geográfico dentro del vasto territorio argentino, al gunos rodeos presentan ciertas diferencias genéticas, sin embargo los mismos forman parte de la raza Criol lo A rgentino y pueden aparearse libremente entre ellos de acuerdo a las necesidades de cada criador.

\section{Características morfológicas de la raza}

\section{Color de capa y piel}

El bovino Criollo Argentino se caracteriza por una gran diversidad de colores de capa, que en cierta manera refleja su riqueza genética y fenotípica. Posee los tres colores de pelo básicos: castaño, negro y blanco; existiendo animales de capa entera, overos, salpicados, manchados y muchas variantes más. Además, la interacción de diferentes genes da lugar a la presencia de animales hoscos y azotados o barcinos. También actúan genes que modifican la intensidad del color castaño, originando capas bayas, doradillas y retintas. A su vez, la pigmentación de las mucosas externas de la piel es variable y permite la presentación de animales con hocico negro, con pintas y sin pigmentación melánica (hocico pardo) (Rabasa et al., 1986).

\section{Cornamenta}

Otra expresión de la diversidad genética y fenotípica de la raza se manifiesta en la existencia de animales criollos con y sin cuernos, reconociendo que la imagen más difundida y popular en esta raza es la de animales con cuernos de una forma muy característica. La 
existencia de animales mochos en el Virreinato del Río de la Plata, previo al ingreso de otras razas bovinas al país, fue descripta por el naturalista español Félix de Azara (Carrazoni, 1998). Testimonios sobre la presencia de animales Criollos sin cuernos, en el año 1790, corresponden a los brindados en la estancia correntina de los Jesuitas “Rincón de Luna”. A demás, fue reportada la existencia de vacas Criollas bayas y mochas en el valle de Punilla, Córdoba, siendo ésta una de las últimas observaciones mencionadas.

La existencia de animales mochos en la raza Criollo Argentino podría deberse al ingreso de animales sin cuernos en el momento de colonización, considerándose esto poco probable. Otra alternativa que permite explicar la presencia de estos animales dentro de la raza es la ocurrencia de mutación durante el tiempo transcurrido luego de su arribo a territorio americano. La aparición de animales mochos tendría una frecuencia de suceder una vez cada 100.000 apareamientos (Bavera, 2000). En este sentido, al evaluar el origen de la mutación mocho en el rodeo de bovinos criollos de INTA Leales se señala la ausencia de una reciente introgresión genética desde otras razas mochas criadas en la región y se apoya la hipótesis de un antiguo origen de la mutación causal de animales sin cuernos (Falomir-Lockhart et al., 2019).

La ausencia de cuernos es un carácter dominante (Holgado y Rabasa, 2001) que no tuvo progreso en cuantía durante los años de selección natural dentro de la raza, sin embargo, actualmente permite al ganadero ampliar de manera sencilla y rápida la frecuencia de animales mochos en el rodeo de acuerdo a su conveniencia comercial y escenario productivo.

\section{M orfometría auricular y cefálica}

El conocimiento de la morfometría de orejas y cabeza en bovinos permite realizar una descripción cuantitativa que favorece la precisión en la definición del patrón racial y la detección de introgresiones genéticas en rodeos generales.

El bovino Criollo Argentino presenta un largo de oreja de $15.3 \mathrm{~cm}$ y un ancho de $9.0 \mathrm{~cm}$ con una relación ancho/ largo de 0.59 (Poli,1993). En el Cuadro 1 se detallan los valores de largo y ancho de orejas, largo y ancho de cabeza y sus relaciones de vacas Criollas adultas con y sin cuernos (Holgado et al ., 2015a).

Cuadro 1. Valores promedios en centímetro (cm) para largo de cabeza (LC), ancho de cabeza (AC), largo de orejas (LO), ancho de orejas (AO) y su relación (LC/ AC, LO/ AO) en vacas Criollo A rgentino adultas mochas y astadas.

\begin{tabular}{lcccccc}
\hline Fenotipo & $\mathrm{LC}(\mathrm{cm})$ & $\mathrm{AC}(\mathrm{cm})$ & $\mathrm{LC} / \mathrm{AC}$ & $\mathrm{LO}(\mathrm{cm})$ & $\mathrm{AO}(\mathrm{cm})$ & $\mathrm{LO} / \mathrm{AO}$ \\
\hline Astado & $48.67 \pm 2.17^{\mathrm{a}}$ & $20.33 \pm 1.19^{\mathrm{a}}$ & $2.40 \pm 0.11^{\mathrm{a}}$ & $15.75 \pm 0.81^{\mathrm{a}}$ & $9.08 \pm 0.52^{\mathrm{a}}$ & $1.74 \pm 0.08^{\mathrm{a}}$ \\
Mocho & $47.81 \pm 1.71^{\mathrm{a}}$ & $20.44 \pm 1.10^{\mathrm{a}}$ & $2.35 \pm 0.17^{\mathrm{a}}$ & $15.83 \pm 0.66^{\mathrm{a}}$ & $9.36 \pm 0.59^{\mathrm{a}}$ & $1.70 \pm 0.11^{\mathrm{a}}$ \\
Promedio & $48.24 \pm 1.95$ & $20.39 \pm 1.14$ & $2.38 \pm 0.14$ & $15.79 \pm 0.74$ & $9.22 \pm 0.56$ & $1.72 \pm 0.10$ \\
\hline
\end{tabular}

aLas diferencias dentro de las columnas están indicadas por diferentes letras $(P<0.05)$.

\section{Longitud de Prepucio}

En Argentina la cría bovina se desarrolla generalmente en ambientes donde predominan pastos altos, fibrosos y arbustos. Estos escenarios predisponen a los machos con prepucios largos y péndulos, a sufrir lesiones que pueden inhabilitarlos para la monta y acortar su vida útil, afectando el costo de producción a través desu reposición.

La longitud del prepucio, cuantificado en centímetros desde la base del abdomen hasta la parte central del orificio de salida de la uretra, de animales Criollo Argentino astados y mochos son mostrados en el Cuadro 2. La longitud media de prepucio de los animales evaluados fue de $7.66 \pm 2.19 \mathrm{~cm}$, no existiendo diferencias estadísticas significativas entre los fenotipos evaluados (Holgado, Rabasa y Ortega, 2019).
Cuadro 2. Longitud de prepucio expresada en $\mathrm{cm}$ de machos de raza Criollo Argentino.

\begin{tabular}{lcc}
\hline Fenotipo & $\begin{array}{c}\text { Número } \\
\text { de machos }\end{array}$ & $\begin{array}{c}\text { Longitud prepucio } \\
(\mathrm{cm})\end{array}$ \\
\hline Astado & 20 & $7.28 \pm 2.21^{\mathrm{a}}$ \\
Mocho & 30 & $7.92 \pm 2.18^{\mathrm{a}}$ \\
Total & 50 & $7.66 \pm 2.19$ \\
\hline
\end{tabular}

aLas diferencias dentro de las columnas están indicadas por diferentes letras $(P<0.05)$

los fenotipos evaluados (Holgado, Rabasa y Ortega, 2019).

En este sentido, Ios machos de raza Criollo Argentino presentan un prepucio de muy corta a corta longitud, lo cual resulta funcionalmente favorable para las condiciones prevalentes en la mayoría de los campos de cría bovina. 


\section{Zoometría y tamaño corporal}

La zoometría estudia las formas de los animales a través de diferentes medidas corporales, permitiendo cuantificar la conformación de los mismos y caracterizar con mayor rigurosidad un recurso genético.
Las medidas corporales de vacas de raza Criollo Argentino varían de acuerdo a su edad hasta estabilizarse en cierto momento, siendo algunas más precoces que otras (Holgado et al., 2015b). La vaca Criollo Argentino es un animal de tamaño corporal relativamente chico, considerándose este un aspecto favorable para las condiciones de producción predominantes en el territorio argentino donde se desarrolla (Cuadro 3).

Cuadro 3. Medidas corporales en hembras de raza Criollo Argentino de acuerdo a su edad en años. (AC=alzada a la cruz, AG=alzada a la grupa, LC=largo corporal, PT=perímetro torácico, PC=perímetro de caña, LC=largo de caña, AAG=ancho anterior de grupa, $\mathrm{APG}=$ ancho posterior de grupa, $\mathrm{LG}$ =largo de grupa y $\mathrm{PCC}=$ peso corporal corregido por condición corporal).

\begin{tabular}{lccccccccccc}
\hline Edad & $\mathrm{n}$ & AC (cm) & AG (cm) & LC (cm) & PT (cm) & PC (cm) & LC (cm) & AAG (cm) & APG (cm) & LG (cm) & PCC (kg) \\
\hline 1 & 9 & $103.2^{\mathrm{d}}$ & $112.8^{\mathrm{d}}$ & $120.9^{\mathrm{d}}$ & $132.2^{\mathrm{e}}$ & $14.8^{\mathrm{b}}$ & $19.1^{\mathrm{e}}$ & $32.1^{\mathrm{e}}$ & $10.7^{\mathrm{d}}$ & $37.3^{\mathrm{d}}$ & $177.4^{\mathrm{e}}$ \\
2 & 7 & $111.1^{\mathrm{c}}$ & $120.3^{\mathrm{c}}$ & $138.7^{\mathrm{c}}$ & $158.1^{\mathrm{d}}$ & $17.0^{\mathrm{a}}$ & $20.7^{\mathrm{dc}}$ & $38.7^{\mathrm{d}}$ & $13.2^{\mathrm{c}}$ & $42.0^{\mathrm{c}}$ & $291.7^{\mathrm{d}}$ \\
3 & 7 & $118.8^{\mathrm{b}}$ & $125.2^{\mathrm{b}}$ & $148.5^{\mathrm{b}}$ & $160.9^{\mathrm{dc}}$ & $17.3^{\mathrm{a}}$ & $21.3^{\mathrm{dcb}}$ & $41.8^{\mathrm{c}}$ & $13.5^{\mathrm{cb}}$ & $47.7^{\mathrm{b}}$ & $350.8^{\mathrm{c}}$ \\
4 & 6 & $126.8^{\mathrm{a}}$ & $133.5^{\mathrm{a}}$ & $158.3^{\mathrm{a}}$ & $167.8^{\mathrm{cb}}$ & $17.5^{\mathrm{a}}$ & $22.5^{\mathrm{ba}}$ & $45.0^{\mathrm{b}}$ & $14.5^{\mathrm{cba}}$ & $51.5^{\mathrm{a}}$ & $399.3^{\mathrm{b}}$ \\
5 & 6 & $124.7^{\mathrm{a}}$ & $130.3^{\mathrm{a}}$ & $163.5^{\mathrm{a}}$ & $172.5^{\mathrm{ba}}$ & $17.6^{\mathrm{a}}$ & $21.2^{\mathrm{dcb}}$ & $46.7^{\mathrm{b}}$ & $15.2^{\mathrm{cba}}$ & $50.7^{\mathrm{a}}$ & $441.3^{\mathrm{a}}$ \\
6 & 6 & $127.7^{\mathrm{a}}$ & $133.3^{\mathrm{a}}$ & $165.2^{\mathrm{a}}$ & $176.7^{\mathrm{a}}$ & $18.2^{\mathrm{a}}$ & $22.6^{\mathrm{ba}}$ & $46.5^{\mathrm{b}}$ & $16.0^{\mathrm{a}}$ & $52.7^{\mathrm{a}}$ & $453.8^{\mathrm{a}}$ \\
7 & 6 & $125.3^{\mathrm{a}}$ & $132.2^{\mathrm{a}}$ & $160.7^{\mathrm{a}}$ & $174.2^{\mathrm{ba}}$ & $17.8^{\mathrm{a}}$ & $21.2^{\mathrm{dcb}}$ & $45.3^{\mathrm{b}}$ & $15.7^{\mathrm{ba}}$ & $51.3^{\mathrm{a}}$ & $443.7^{\mathrm{a}}$ \\
8 & 2 & $126.0^{\mathrm{a}}$ & $133.0^{\mathrm{a}}$ & $166.0^{\mathrm{a}}$ & $179.0^{\mathrm{a}}$ & $18.0^{\mathrm{a}}$ & $20.5^{\mathrm{ed}}$ & $50.5^{\mathrm{a}}$ & $15.0^{\mathrm{cba}}$ & $53.0^{\mathrm{a}}$ & $482.5^{\mathrm{a}}$ \\
9 & 5 & $123.2^{\mathrm{ab}}$ & $131.2^{\mathrm{a}}$ & $162.0^{\mathrm{a}}$ & $176.6^{\mathrm{a}}$ & $17.9^{\mathrm{a}}$ & $22.8^{\mathrm{a}}$ & $47.2^{\mathrm{b}}$ & $15.6^{\mathrm{ba}}$ & $50.2^{\mathrm{ba}}$ & $463.0^{\mathrm{a}}$ \\
10 & 4 & $125.0^{\mathrm{a}}$ & $139.5^{\mathrm{a}}$ & $161.5^{\mathrm{a}}$ & $173.0^{\mathrm{ba}}$ & $18.1^{\mathrm{a}}$ & $22.3^{\mathrm{cba}}$ & $46.5^{\mathrm{b}}$ & $14.8^{\mathrm{cba}}$ & $52.8^{\mathrm{a}}$ & $456.3^{\mathrm{a}}$ \\
\hline
\end{tabular}

a, b, c Las diferencias entre las edades dentro de las columnas están indicadas por diferentes letras $(P<0.05)$.

Con respecto a los machos, los toros adultos presentan un peso corporal medio de $685 \pm 28 \mathrm{~kg}$ considerando una condición corporal (CC) media de 3.5 puntos (escala 1 a 5), una alzada a la cruz de $138.2 \pm 2.5 \mathrm{~cm}$ y una circunferencia escrotal de $37.5 \pm 2.0 \mathrm{~cm}$. Para similar condición corporal el toro Criollo es 1.5 veces más pesado que la hembra (H olgado y Ortega, 2015c).

En ganadería de cría, el tamaño corporal es una característica importante dado que el principal costo para la producción de un ternero es el mantenimiento de la vaca. Esto puede ser considerado como un costo fijo, al cual se suman los costos de gestación y lactancia para determinar un costo total. Además, el tamaño corporal de las hembras interactúa con el contexto nutricional e influye en la eficiencia reproductiva del rodeo.

El peso medio de la vaca adulta de raza Criollo Argentino es de $430 \mathrm{~kg}$ a condición corporal media de 3 puntos. Las hembras con y sin cuernos no difieren estadísticamente en su tamaño corporal, teniendo la condición corporal un efecto significativo $(P<0.05)$ en las variaciones de peso de los vientres (Cuadro 4). En este sentido, el peso de la vaca aumenta junto con su condición corporal, siendo el incremento de $58.4 \mathrm{~kg}$ por unidad de CC (Holgado y Ortega, 2013). Otras razas Criollas presentan un peso adulto concordante al informado para el Criollo Argentino (De Alba, 2011; Camargo, 1991; Mariante, 1993; Wilkins y Rojas, 1993).

Cuadro 4. Condición corporal (escala 1 a 5) y peso (kg) de la vaca Criollo Argentino adulta.

\begin{tabular}{cc}
\hline Condición Corporal & Peso vaca adulta $(\mathrm{kg})$ \\
\hline 1 & 313 \\
2 & 372 \\
3 & 430 \\
4 & 489 \\
5 & 547 \\
\hline
\end{tabular}

\section{Aptitud Lechera}

La producción de leche en razas bovinas de carne puede variar entre 700 y 1500 litros por lactancia, siendo el costo animal para producir un litro de leche de aproximadamente $1 \mathrm{M}$ cal de energía metabólica; lo que representa un $20-25 \%$ del total requerido anualmente por un vientre.

Existe poca información acerca de la producción de leche en la raza Criollo Argentino. Vacas adultas seleccionadas para dar los mejores pesos de destete de terneros, tienen una producción de 957 litros de leche por lactancia. A nivel de rodeo, esta producción de leche no supera los 4 litros/ día. En este sentido, la lactancia en destetes a 210 días de vida del ternero, alcanzaría un valor de 840 litros (Rabasa et al., 2005). 
En base a esta información, la vaca Criolla presenta una aptitud lechera moderada, siendo suficiente para criar sin problemas a su ternero. Las ganancias de peso predestete de las crías podrán variar entre 500 y 700 gramos por día, generando pesos de destete de entre 133 a 176 kg a los 210 días de vida de las crías.

La producción de leche en esta raza permite lograr un equilibrio entre la eficiencia de producción y la eficiencia reproductiva, consiguiendo un mayor número de terneros de menor peso, en concordancia a los escenarios ambientales en los que se desarrolla.

\section{Longevidad}

La vaca Criolla es un animal longevo, que puede permanecer en el rodeo de cría hasta edades avanzadas (12-16 años). Si se considera el desgaste de las piezas dentarias como criterio de permanencia de la hembra en el sistema productivo, la longitud de la corona en Criollo Argentino disminuye a razón de 1.15 $\mathrm{mm} /$ año (Longitud de corona $=23.58-1.15 X$ ). De esta manera, una vaca alcanza la edad de 12 años con un 54 $\%$ de su longitud de corona inicial y 14 años con un 29 $\%$ (Holgado et al., 2016b).

Además, la longevidad puede ser explicada por la dureza del esmalte dental. Novillos Criollo Argentino Patagónico presentan una mayor dureza de esmalte dental que aquellas cruzas índicas (Fernández et al., 2011).

\section{Resistencia a enfermedades y factores ambientales}

Dentro de Bos taurus, el bovino Criollo Argentino demuestra ser resistente a diversas enfermedades y factores ambientales. Muchos de estos comunicados surgen a partir de observaciones de campo que indican su resistencia a sarna, calidad del agua de bebida, altas y bajas temperaturas, entre otras. Asociado a la conformación ocular y pigmentación de mucosas, el Criollo posee resistencia genética a queratoconjuntivitis (Sal Paz de y Holgado, 1995). Además, la raza es resistente a la carga de garrapata común del bovino, lo que permite proponerlo como una alternativa atractiva para el desarrollo de la ganadería en las regiones endémicas para el mencionado ectoparásito (Ortega y Holgado, 2017).

\section{Características productivas}

\section{Producción en cría}

Es conocido que la producción de cría bovina de cualquier raza o cruza depende de un componente genético, otro ambiental y de la interacción entre ambos. Además, se considera que la fertilidad es de gran importancia y ha sido descuidada (Rabasa, 1986). No hay dudas que los animales no sólo deben nacer, sino también sobrevivir. Por lo tanto, la facilidad de parto y la capacidad de supervivencia hasta el destete o hasta la edad de faena, son factores fundamentales en la producción de carne. La viabilidad depende en esencia de la adaptación, ya que si el animal no puede afrontar los desafíos del ambiente o de su propia fisiología es razonable suponer que le fal ta adaptación.

El ganado Criollo Argentino posee una producción en cría que logra superar a la alcanzada por razas como Nelore y Hereford en un ambiente subtropical. En el trabajo llevado a cabo por Sal Paz (1986) los tres genotipos tuvieron un nivel de fertilidad similar, pero los valores de mortandad de terneros difirieron significativamente (Holgado, Rabasa de Sal Paz y Sal Paz, 1988), resultando la raza Criollo Argentino la más productiva en términos de $\mathrm{kg} / \mathrm{ha} /$ año (Criollo Argentino 93.2; Hereford 68.5; Nelore 89.5 $\mathrm{kg} / \mathrm{ha} / \mathrm{año}$ ).

La Unidad Experimental de INTA Leales (Tucumán) representa un ambiente subtropical- subhúmedo con estación seca (Torres Bruchman, 1977). Las Iluvias alcanzan un valor promedio anual de $910 \pm 228 \mathrm{~mm}$, concentrándose el $90 \%$ de las mismas entre los meses de octubre y abril. La evapotranspiración es de 900 $\mathrm{mm} /$ año y la ocurrencia de heladas posee una frecuencia media de 16 al año (mayo a septiembre). La temperatura media anual es de $19{ }^{\circ} \mathrm{C}$, siendo la máxima media de $25^{\circ} \mathrm{C}$ en el mes de enero y la mínima media de $13^{\circ} \mathrm{C}$ en julio. La base de alimentación forrajera corresponde a pasturas megatérmicas implantadas (Brachiaria brizantha, Chloris gayana, M egathyrsus maximus, Cynodon dactylon), con alta capacidad de carga aunque variable según la calidad de los suelos ( 0.5 a 1.5 vacas/ ha).

En el Cuadro 5 son presentados los resultados productivos de 10 años del rodeo de bovinos Criollo Argentino perteneciente al INTA Leales (Holgado y Ortega, 2019). 
Cuadro 5. Comportamiento productivo del rodeo Bovino Criollo del INTA Leales (Tucumán). Período de años 2006 a 2016.

\begin{tabular}{|c|c|c|c|c|c|c|c|c|c|c|c|c|c|}
\hline AÑO & 2006 & 2007 & 2008 & 2009 & 2010 & 2011 & 2012 & 2013 & 2014 & 2015 & 2016 & media & $\mathrm{DE}$ \\
\hline Nro vientres & 85 & 89 & 88 & 116 & 97 & 91 & 98 & 105 & 115 & 117 & 111 & 101.1 & 12.2 \\
\hline Preñez ( \%) & 82.4 & 78.7 & 80.7 & 87.1 & 85.6 & 94.5 & 90.8 & 95.2 & 93.9 & 98.3 & 94.6 & 89.2 & 6.7 \\
\hline Abortos ( \%) & 0 & 5.7 & 1.4 & 0 & 2.4 & 0 & 1.1 & 3 & 0.9 & 1.7 & 2.9 & 1.7 & 1.7 \\
\hline Parición ( \%) & 82.4 & 74.2 & 79.5 & 87.1 & 83.5 & 94.5 & 89.8 & 92.4 & 93 & 96.6 & 91.9 & 87.7 & 7 \\
\hline \multicolumn{14}{|l|}{ Muertes nac-dtt } \\
\hline (\%) & 7.1 & 4.5 & 7.1 & 5 & 3.7 & 1.2 & 6.8 & 6.2 & 0.9 & 4.4 & 2 & 4.5 & 2.3 \\
\hline Destete ( \%) & 76.5 & 70.8 & 73.9 & 82.8 & 80.4 & 93.4 & 83.7 & 86.7 & 92.2 & 92.3 & 90.1 & 83.9 & 7.9 \\
\hline \multicolumn{14}{|l|}{ Peso de } \\
\hline destete real (kg) & 140.5 & 130.3 & 133.6 & 147.3 & 160.8 & 174.9 & 156.3 & 166.3 & 165.4 & 149.7 & 151.1 & 152.4 & 14 \\
\hline kg/ vaca/ año & 107.4 & 92.2 & 98.7 & 121.9 & 129.3 & 163.4 & 130.8 & 144.1 & 152.5 & 138.2 & 136.1 & 127.8 & 2.1 \\
\hline
\end{tabular}

El buen desempeño productivo de vacas Criollas es logrado también en ambientes áridos como el que representa la unidad del INTA Chamical, ubicado en la provincia de La Rioja; con lluvias de $300 \pm 100$ $\mathrm{mm} /$ año concentradas entre los meses de noviembre a marzo y una temperatura media anual de $19{ }^{\circ} \mathrm{C}$. En esta región, definida como Chaco árido, la vegetación se compone de estratos (arbóreo, arbustivo y graminoso). El estrato graminoso es cuantitativamente el más importante en la dieta del vacuno, presentando una marcada estacionalidad en cuanto a crecimiento y calidad; y definiendo una receptividad ganadera muy baja (1 vaca cada 10 a 30 ha). En el Cuadro 6 se muestran los resultados productivos correspondientes a ocho años de evaluación de los vientres del rodeo de bovinos Criollos del INTA Chamical de capa entera doradilla en comparación al rodeo general de animales Criollos pertenecientes a la unidad de investigación (Ferrando, 1986).

Cuadro 6. Índices productivos de vientres Criollos pertenecientes al rodeo general y al rodeo de capa entera doradilla del INTA Chamical (La Rioja), promedio de los años 1997 a 2005.

\begin{tabular}{lcc}
\hline Índices productivos & Rodeo Criollo general & Rodeo Criollo capa entera \\
\hline Preñez (\%) & $90.6 \pm 8.8$ & $90.4 \pm 5.6$ \\
Parición (\%) & $89.9 \pm 8.7$ & $89.2 \pm 5.1$ \\
Destete (\%) & $89.1 \pm 8.4$ & $88.6 \pm 4.8$ \\
Peso de nacimiento $(\mathrm{kg})$ & $27.9 \pm 1.2$ & $27.5 \pm 0.9$ \\
Peso de destete $(\mathrm{kg})$ & $158.0 \pm 20.0$ & $155.0 \pm 19.0$ \\
Ganancia de peso $(\mathrm{kg} / \mathrm{dia})$ & $0.619 \pm 0.067$ & $0.607 \pm 0.045$ \\
kg ternero/ vientre & $140.0 \pm 19.0$ & $137.0 \pm 17.0$ \\
\hline
\end{tabular}

Asimismo, en los Llanos riojanos la raza Criollo Argentino demostró un buen desempeño productivo en comparación con animales de raza Angus (Ferrando et al., 2006).

\section{Peso al Nacer, al destete y aumento de peso predestete}

El peso al nacimiento de los terneros es importante porque guarda relación con el tamaño adulto y la facilidad de parto. Mientras que el peso al destete, junto con el porcentaje de destete, determinan los kilogramos de ternero producidos anualmente. A mbas variables son afectadas por efectos ambientales tales como año de nacimiento, edad de la madre y sexo de la cría (Holgado, Ortega y Cantarella, 2019). En el Cuadro 7 se presentan los valores de peso de nacimiento, destete y ganancia de peso predestete de terneros de vacas Criollas de diferente edad al parto. Los terneros machos son de mayor tamaño corporal que las hembras, resultando un $2.3 \%$ más pesados al nacimiento y un $8.8 \%$ al destete (Cuadro 8). Los pesos de destete obtenidos en ganado Criollo son, en general, livianos; lo que evidencia la moderada producción de leche de la vaca y los menores requerimientos para el vientre, como fue explicado anteriormente.

Cuando la producción de leche de la madre es moderada y la calidad de las pasturas es mediana, la suplementación al pie de la vaca tiene un impacto importante sobre la ganancia de peso predestete y en consecuencia el peso de destete. Durante los dos primeros meses de vida el ternero cubre sus requerimientos nutricionales casi exclusivamente con leche materna. A partir del tercer mes la leche comienza a ser insuficiente y el aporte del forraje consumido adquiere relevancia. En el Cuadro 9 se detalla el efecto de la suplementación al pie de la madre, con un balanceado comercial, sobre el 
crecimiento de terneros Criollo (Holgado et al., 2019). Los animales evaluados tuvieron ganancias de peso similares previo al inicio de suplementación ( $642 \mathrm{~g} / \mathrm{d}$ ). Durante el período de ensayo, las ganancias de peso de los terneros con acceso al suplemento fueron un 75 $\%$ más altas que la de aquellos que no lo recibieron; permitiendo incrementar en un $35 \%$ la ganancia de peso desde el nacimiento hasta el destete.

Cuadro 7. Peso al nacer, al destete ajustado a 205 días de vida y ganancia diaria de peso predestete (GDP) para terneros de vacas Criollo A rgentino de diferentes edades.

\begin{tabular}{ccccc}
\hline Edad vaca (años) & $\mathrm{N}^{\circ}$ de animales & Peso nacimiento $(\mathrm{kg})$ & Peso destete $(\mathrm{kg})$ & $\mathrm{GDP}(\mathrm{g} / \mathrm{d})$ \\
\hline 2 & 46 & $20.2^{\mathrm{c}}$ & $94.4^{\mathrm{c}}$ & $361.7^{\mathrm{c}}$ \\
4 & 41 & $27.1^{\mathrm{ab}}$ & $144.3^{\mathrm{b}}$ & $571.6^{\mathrm{b}}$ \\
5 & 42 & $27.5^{\mathrm{ab}}$ & $157.6^{\mathrm{ab}}$ & $634.9^{\mathrm{ab}}$ \\
6 & 36 & $28.2^{\mathrm{ab}}$ & $159.5^{\mathrm{ab}}$ & $640.6^{\mathrm{ab}}$ \\
$7-10$ & 56 & $27.9^{\mathrm{ab}}$ & $166.3^{\mathrm{a}}$ & $675.3^{\mathrm{a}}$ \\
$11-12$ & 19 & $27.7^{\mathrm{ab}}$ & $159.5^{\mathrm{a}}$ & $643.3^{\mathrm{a}}$ \\
$13-14$ & 15 & $28.3^{\mathrm{a}}$ & $156.4^{\mathrm{ab}}$ & $625.2^{\mathrm{ab}}$ \\
$15-16$ & 6 & $26.9^{\mathrm{b}}$ & $150.1^{\mathrm{b}}$ & $601.5^{\mathrm{b}}$ \\
\hline
\end{tabular}

a, b, c Las diferencias entre las edades dentro de las columnas están indicadas por diferentes letras $(P<0.05)$

Cuadro 8. Peso al nacer, al destete ajustado a 205 días de vida y ganancia diaria de peso predestete (GDP) de terneros machos y hembras de la raza Criollo A rgentino.

\begin{tabular}{lcccc}
\hline Sexo & $\begin{array}{c}\mathrm{N}^{\circ} \text { de } \\
\text { animales }\end{array}$ & $\begin{array}{c}\text { Peso } \\
\text { nacimiento } \\
(\mathrm{kg})\end{array}$ & $\begin{array}{c}\text { Peso } \\
\text { destete } \\
(\mathrm{kg})\end{array}$ & $\begin{array}{c}\text { GDP } \\
(\mathrm{g} / \mathrm{d})\end{array}$ \\
\hline Macho & 126 & $27.0^{\mathrm{a}}$ & $154.8^{\mathrm{a}}$ & $0.623^{\mathrm{a}}$ \\
Hembra & 135 & $26.4^{\mathrm{b}}$ & $142.3^{\mathrm{b}}$ & $0.565^{\mathrm{b}}$ \\
\hline
\end{tabular}

a, b Las diferencias dentro de las columnas están indicadas por diferentes letras $(P<0.05)$

Cuadro 9. Peso corporal al inicio y final del período de suplementación al pie de la vaca y ganancias diarias de peso (GDP) de terneros Criollo Argentino sin y con acceso a alimento balanceado ad libitum.

\begin{tabular}{lcc}
\hline Variables & $\begin{array}{c}\text { Peso Terneros } \\
\text { sin suplemento } \\
(\mathrm{kg})\end{array}$ & $\begin{array}{c}\text { Peso Terneros } \\
\text { con suplemento } \\
(\mathrm{kg})\end{array}$ \\
\hline $\begin{array}{l}\text { Peso inicio } \\
\text { ensayo }(\mathrm{kg})\end{array}$ & $92.0^{\mathrm{a}}$ & $93.9^{\mathrm{a}}$ \\
$\begin{array}{l}\text { Peso final } \\
\text { ensayo }(\mathrm{kg})\end{array}$ & $154.6^{\mathrm{a}}$ & $203.5^{\mathrm{b}}$ \\
$\begin{array}{l}\text { GDP durante } \\
\text { el ensayo }(\mathrm{g} / \mathrm{d})\end{array}$ & $585.0^{\mathrm{a}}$ & $1024.0^{\mathrm{b}}$ \\
$\begin{array}{l}\text { GDP nacimiento } \\
\text { a dtte }(\mathrm{g} / \mathrm{d})\end{array}$ & $616.0^{\mathrm{a}}$ & $831.0^{\mathrm{b}}$
\end{tabular}

$\mathrm{a}, \mathrm{b}$ Las diferencias entre las columnas están indicadas por diferentes letras $(P<0.05)$

El consumo de suplementación a voluntad del ternero tiene un impacto importante en la ganancia de peso y en consecuencia en el peso al destete de animales de la raza Criollo Argentino. Un consumo promedio de 1.87 $\mathrm{kg} / \mathrm{cab} /$ día de suplemento genera un incremento en la ganancia diaria de peso del orden de los 439 gramos, logrando una eficiencia de conversión aparente de 4.26 $\mathrm{kg}$ de alimento por $\mathrm{kg}$ de ganancia de peso. Estos valores indican la posible conveniencia de incorporar esta práctica, especialmente en crías de vacas de ler parto.

El fenotipo de la madre, mocha o astada, no tiene efecto estadísticamente significativo sobre el peso de nacimiento y la ganancia de peso predestete de terneros de raza Criolla (Holgado et al., 2016a).

\section{Precocidad sexual}

La edad de ingreso de la vaquillona al primer servicio tiene importancia productiva y económica y debe ser contemplada para la intensificación de los sistemas de cría en búsqueda de lograr una mayor eficiencia. Para que una vaquillona ingrese a servicio a los 15 meses de edad es necesario contar con un recurso genético precoz, capaz de al canzar la pubertad antes de esa edad. El componente genético debe acompañarse de una nutrición adecuada para tal finalidad (Ferraz, 2018). La pubertad se logra cuando la vaquillona alcanza el $65 \%$ de su peso adulto (Brody, 1964), aunque en vaquillonas para carne puede alcanzarse cuando logra un $50 \%$ del peso adulto (Roy, 1974). En la hembra existe un peso crítico a partir del cual se disparan una serie de eventos hormonales endocrinos que inducen la llegada de la pubertad (Gree et al., 1983; Mc Andrews et al., 1993; Adam y Robinson, 1994; Grajales, Hernández y Prieto, 2006) y el desarrollo de una actividad sexual continua (A beygunawardena y Dematawewa, 2004).

El crecimiento de vaquillonas de raza Criollo Argentino, con un peso de nacimiento promedio de $26.97 \pm 2.29 \mathrm{~kg}$ es variable de acuerdo a la época de parición y a las ganancias de peso predestete (Holgado, Fernández y Ortega, 2017c). Las ganancias de peso luego del destete de las hembras y hasta su ingreso a servicio a los 15 meses de edad deberían tener valores 
de entre 600 a $700 \mathrm{~g} / \mathrm{d}$, para lograr un peso del 60-65 $\%$ del peso adulto de una vaca Criolla con condición corporal (CC) de 3.5 (escala 1 a 5).

El peso corporal crítico de inicio de pubertad en vaquillonas de raza Criollo Argentino puede ser considerado entre los 240 y $250 \mathrm{~kg}$ (Holgado, Fernández y Ortega, 2017a), con una mayor proporción y rapidez de preñez a medida que aumenta el mismo.

Los pesos de nacimiento de crías de vaquillonas Criollas con servicio a los 15 meses de edad difieren de acuerdo al sexo, logrando los machos un peso promedio de $21.4 \mathrm{~kg}$ y las hembras $19.5 \mathrm{~kg}$; sin observarse problemas de distocia al parto a pesar del limitado desarrollo corporal de algunos vientres. La ganancia de peso predestete promedio de estas crías es de $407 \pm 107 \mathrm{~g} /$ día (Holgado, Fernández y Ortega, 2017b), incrementándose este valor con el aumento de peso de la vaquillona. En los primeros meses de vida de los terneros se produce un elevado porcentaje de pérdidas (18.67\%), posiblemente relacionado al poco desarrollo mamario y consecuente baja producción de leche de las vaquillonas en general.

En virtud de no afectar la fertilidad futura de vientre es recomendable lograr un peso de ingreso a servicio a los 15 meses de edad de $290 \mathrm{~kg}$. El crecimiento predestete es un componente importante en relación al peso que tendrá la vaquillona a su ingreso a servicio, siendo clave obtener un buen peso de destete para arrancar con una ternera bien desarrollada y disminuir las exigencias durante el posdestete.

\section{Comportamiento reproductivo}

El intervalo entre partos ha sido señalado como uno de los mejores indicadores de la eficiencia reproductiva de los vientres dentro de los sistemas de producción de cría bovina, siendo variable entre individuos debido a diferencias genéticas y ambientales.

En el Cuadro 10 se describe la influencia de la condición corporal sobre el intervalo entre partos de vacas Criollo Argentino de entre 5 y 17 años de edad pertenecientes al rodeo de INTA Leales, con servicio estacionado entre los meses de diciembre y febrero. El intervalo entre partos logrado en este rodeo es de $370.13 \pm 23.45$ días, presentando una correlación negativa con la condición corporal de la hembra en su ingreso a servicio (Intervalo entre partos $=426.94$ $21.25 \mathrm{CC}$ a entrada a servicio, $\mathrm{P}<0.01$ ). En este sentido, una condición corporal de 3 puntos (escala 1 a 5) permite reducir el intervalo entre partos a 363.19 días (Holgado et al., 2019).

Cuadro 10. Intervalo entre partos (IEP) y condición corporal al parto e ingreso a servicio (CC = escala 1 a 5) de vacas adultas de raza Criollo Argentino.

\begin{tabular}{lcccc}
\hline $\begin{array}{l}\text { Año de } \\
\text { parición }\end{array}$ & $\begin{array}{c}\text { Número } \\
\text { dehembras }\end{array}$ & Intervalo entre partos & CC al parto & CC ingreso a servicio \\
\hline 2013 & 52 & $390.2^{\mathrm{a}}$ & 2.56 & $2.18^{\mathrm{a}}$ \\
2014 & 72 & $348.2^{\mathrm{c}}$ & 2.90 & $3.09^{\mathrm{c}}$ \\
2015 & 66 & $371.3^{\mathrm{b}}$ & 2.84 & $2.69^{\mathrm{b}}$ \\
2016 & 59 & $373.4^{\mathrm{b}}$ & 2.86 & $2.72^{\mathrm{b}}$ \\
2017 & 59 & $374.6^{\mathrm{b}}$ & 2.72 & $2.53^{\mathrm{b}}$ \\
M edia & & $370.13 \pm 23.5$ & $2.79 \pm 0.29$ & $2.67 \pm 0.49$ \\
a,b, Las diferencias entre los años de parición dentro de las columnas están indicadas por diferentes letras $(\mathrm{P}<0.01)$
\end{tabular}

El buen desempeño reproductivo de la raza, determinado por un elevado porcentaje de preñez y un corto intervalo entre partos, se sustenta en dos aspectos fundamentales: lograr una buena condición corporal de las hembras en su ingreso a servicio y tener una elevada proporción de vacas paridas temprano dentro del periodo de ocurrencia de partos. En este sentido, en el Cuadro 11 se detalla el porcentaje de preñez logrado por los vientres Criollos en función de su condición corporal al inicio de servicio a lo largo de distintos años, con una concentración de nacimientos en el primer mes de parición (69\%) (Holgado et al., 2019).

\section{C recimiento post destete}

El crecimiento posdestete de los animales es influenciado por factores como la alimentación y la genética, a través de la tasa de crecimiento, composición de la ganancia de peso y el tamaño corporal adulto. En la medida que una raza o cruza posea un mayor tamaño adulto, mayor será su peso corporal y ganancia de peso en todas las edades; aunque de menor precocidad. En este sentido, para una misma edad, un animal de mayor tamaño corporal alcanzará un menor porcentaje de peso en relación al logrado a su edad adulta. Animales de 
razas de menor tamaño corporal acumularán un mayor contenido de grasa, resultando menos eficiente en su conversión de alimento. Sin embargo, esto no es así cuando las comparaciones se realizan a igual grado degordura.

Novillos de raza Criollo Argentino logran ganancias diarias de peso similares a la raza sintética Braford (Garriz et al., 1984), con valores que alcanzan los 1390 g/ d (Zimerman et al., 2019). Además, el crecimiento y desempeño en invernada de novillos Angus, Hereford, Shorthorn, Beefmaster y cruzas (Filial 1: Criollo Argentino x Británicas) poseen similar aptitud al logrado por la raza Criollo Argentino (Garriz et al., 1998d). Terneros Criollos recriados sobre Cenchrus ciliaris logran superar en un $13 \%$ a terneros Angus en producción de carne total y ganancia diaria de peso (Namur et al., 2004)

\section{Producto final}

Existe unanimidad de opinión en cuanto a que la evaluación comercial en pie de Criollo Argentino no resulta favorable debido a la heterogeneidad de pelajes, presencia de grandes cuernos y conformación menos "carnicera" (más longilíneos y angulosos) respecto a otras razas. De igual manera, la tipificación de la res en base a conformación no favorece al Criollo, por tratarse de animales menos redondeados y compactos (Garriz et al., 1986; Garriz et al., 1991; Gallinger, Garriz y Mezzadra, 1995).

Las diferencias en conformación están asociadas comúnmente a diferentes rendimientos carniceros de las reses. Sin embargo, los animales de raza Criollo Argentino, engordados en iguales condiciones, presentan reses con similares porcentajes de músculo, grasa y hueso que la raza Braford de mejor conformación ( $69 \%$, $13 \%, 16 \%$ y $63 \%, 19 \%$ y $16 \%$ para Criollo y Braford respectivamente). Además, se indica que Criollo y Braford poseen igual porcentaje de cortes de cuarto pistola (Garriz et al., 1991). Tanto Criollo Argentino, como sus cruzas con razas británicas, tienen igual aptitud carnicera que novillos Angus, Hereford, Shorthorn y Beefmaster para producir cortes de exportación (Hilton) (Gallinger et al., 1998a).

El aumento de peso vivo es acompañado por el incremento en kilos de músculo ( $r 0.92 \mathrm{P}<0.05)$ en novillos Angus, Hereford, Shorthorn, Beefmaster y Criollo Argentino; sin embargo el porcentaje de músculo disminuye $(r-0.18 \mathrm{P}<0.05)$. En promedio, el porcentaje de músculo de estos biotipos es $33 \%$, logrando la grasa y el hueso valores de $11 \%$ y $9 \%$ respectivamente y no habiendo diferencias estadísticas significativas $(P<0.05)$ entre las razas respecto al peso vivo de faena, ni a la proporción de los principales componentes corporales que determinan el rendimiento carnicero (Garriz et al ., 1998d).

La carne obtenida de animales Criollo Argentino es de similar terneza y cualidades a la de otras razas y cruzas (Zimerman et al., 2019). Con el incremento de edad del animal, el peso vivo y la deposición de grasa aumentan y disminuyen los promedios de resistencia al corte (WB, lb). El nivel de grasa en la res influye en el efecto del frío sobre el acortamiento de la fibra muscular y la terneza. La faena de novillos Criollos a los 18, 24 y 30 meses de edad logra valores de resistencia al corte de $8.6,6.9$ y $5.7 \mathrm{lb}$ respectivamente, sin diferir significativamente con otros biotipos (Garriz et al., 1998c). De esta manera, la raza Criollo Argentino y sus cruzas no comprometen la terneza de la carne obtenida, como así tampoco las características sensoriales y reológicas (Gallinger et al., 1998).

Cuadro 11. Porcentaje de vientres según su condición corporal al inicio de servicio (CC, escala 1 a 5) y porcentaje de preñez logrado entre los años 2011 a 2018 en INTA Leales.

\begin{tabular}{lccccc}
\hline Año & CC 1.5-2.0(\%) & CC 2.5(\%) & CC 3.0 (\%) & CC 3.5-4.0(\%) & Preñez (\%) \\
\hline 2011 & 8 & 33 & 24 & 35 & 94.5 \\
2012 & 1 & 25 & 61 & 13 & 90.8 \\
2013 & 19 & 22 & 26 & 33 & 95.2 \\
2014 & 43 & 27 & 22 & 8 & 93.9 \\
2015 & 6 & 26 & 34 & 34 & 98.3 \\
2016 & 12 & 50 & 35 & 3 & 94.6 \\
2017 & 1 & 74 & 14 & 11 & 95.1 \\
2018 & 1 & 60 & 12 & 27 & 91.7 \\
Media & 11.4 & 39.6 & 28.5 & 20.5 & 94.3 \\
\hline
\end{tabular}

La raza bovina Criollo Argentino es un recurso zoogenético ampliamente caracterizado desde diferentes aspectos, con capacidad para adaptarse a diferentes ambientes agroecológicos y productivos.
Su tamaño corporal relativamente chico y su moderada aptitud lechera le permiten desarrollarse bajo condiciones nutricionales severas, logrando adecuados niveles de fertilidad. 
Esta raza bovina presenta precocidad sexual y sería factible implementar el primer servicio de vaquillonas a los 15 meses de edad sin que se presenten problemas de distocia al parto. Sin embargo, la baja aptitud lechera de esta categoría determina la conveniencia de utilizar suplementación del ternero al pie de la vaca para lograr destetes de mayor peso y disminuir la tasa de pérdida de crías.
Adicionalmente, las reses de animales Criollo Argentino obtenidas en faena presentan una performance y rendimiento carnicero similares a otras razas carniceras producidas bajo iguales condiciones.

Actualmente el Criollo Argentino posee un espacio de mayor protagonismo dentro de la ganadería bovina argentina, tanto como raza pura como en programas de cruzamientos. No obstante, su estudio, difusión y utilización aún constituyen la mejor estrategia de conservación.

\section{Literatura Citada}

A beygunawardena, H. y C.M.B. Dematawena. 2004. Pre-pubertal and postpartum anestrus in tropical Zebu cattle. Animal reproduction Science 82 (83): 373-387.

https:/ / doi.org/ 10.1016/ j.anireprosci.2004.05.006

Adam, C.L. y J.J. Robinson. 1994. The role of nutrition and photoperiod in the timing of puberty. Proceedings N utrition Society 53:89-102.

Bavera, G.A. 2000. Cuernos, mochos, tocos y botones. Cursos de Producción Bovina de Carne. FAVUNRC. www.produccion-animal.com.ar

Brody, S. 1945. Bioenergetics and growth with special reference to the efficiency complex in domestic animals. First published: Reinhold, NY. Reprinted: Hafner Publishing Co., Inc. NY. 1964.

Camargo Almeida, E.A. 1991. Ganado Criollo en Brasil, origen e introducción. Características zootécnicas. Algunos resultados preliminares. En Ganado Bovino Criollo, tomo 2, pág 65-76. Buenos Aires, Editorial Orientación gráfica.

Carrazoni, J.A. 1998. El Bovino Criollo. Academia Nacional de Agronomía y Veterinaria. Tomo LII, No 16, Bs As, Argentina. ISSN 0327-8093.

De Alba Martínez, J. 2011. El libro de los Bovinos Criollos de América. Ed. Papiro Omega, S.A. de C.V. México.

Falomir-Lockhart, A.H., Ortega Masague, M.F., Rudd Garcés, G., Zappa, M.E., Peral García, P., Morales, H.F, Holgado, F.D, Rogberg Muñoz, A. y G. Giovambattista. 2019. Polledness in Argentinean Creole, five centuries surviving. Animal Genetics https:/ / onlinelibrary.wiley.com

Fernández, E. T., A bbiati, N., Cabrera, J., R. Martínez. 2011. Microdureza del esmalte dental en incisivos centrales permanentes de dos genotipos bovinos. Rev. MVZ Córdoba 16 (1):2310-2316.

Ferrando, C., Paloma, E., Namur, P. y D. Leguiza. 2006. Ganado bovino Criollo Argentino y Aberdeen Angus en los Llanos de La Rioja. Resultados de 11 años de evaluación en sistemas de cría. Ediciones INTA, A rgentina. ISSN 1669-323X.
Ferrando, C.A. 1986. La raza Criolla en La Rioja: algunos resultados preliminares. En Ganado Bovino Criollo, tomo 1, pág 17-24.

Ferraz, M.V.C., Pires, A.V., Santos, M.H., Silva, R.G., Oliveira, G.B., Polize, D.M.I, Biehl M.V., Sartor, R. y G.P. Nogueira. A combination of nutrition and genetics is able to reduce age at puberty in Nelore heifers to below 18 months. Animal, Vol 12, Issue 3, 2018, pág 569-574, ISSN 1751-7311, https:/ / doi.org/ 10.1017/ S1751731117002464.

Gallinger, M.M., Garriz, C.A., Suarez, V., Picallo, A.B. y y M. Bussetti. 1998. Evaluación sensorial y reológica de la calidad de carne en novillos puros y cruzas Criollo Argentino. Rev. Arg. Prod. Anim. 18 (Supl.)1:269-270.

Gallinger, M.M.; Garriz, C.A., y C. Mezzadra 1995. Novillos Criollo Argentino y A. Angus en dialélico completo: Evaluación de calidad de carne. Ganado Bovino Criollo, Tomo 4, pág 165-170. Bs. As., Ed. Orientación Gráfica.

Garriz, C.A. 1986. Calidad de res y carne en novillos de raza Criolla Argentina y sus cruzas con Aberdeen Angus. En Ganado Bovino Criollo. Tomo 1. pág 2544. Buenos Aires, Editorial Orientación gráfica.

Garriz, C.A. M., Gallinger, Picallo, A., Bussetti, M y Suárez V. 1998c. Calidad de carne en novillos puros y cruzas Criollo Argentino. Rev. Arg. Prod. Anim. 18 (Supl.)1: 268-269.

Garriz, C.A., Carduza, F., Busetti, M.R., Suárez, B. y Rivera, M.I. 1998d. Crecimiento e invernada de novillos puros y cruzas Criollo Argentino. Rev. Arg. Prod. Anim. 18 (Supl. 1): 282-283.

Garriz, C.A., Gallinger, M., Romero, N., Suárez, V., Bussetti, M y F. Carduza. 1998b. Cortes "Hilton" de novillos puros y cruzas Criollo Argentino. Rev. Arg. Prod. Anim. 18 (Supl. 1): 262-263.

Garriz, C.A., Sal Paz, F., Zamorano, M. y Gallinger M.M. 1991. Rendimiento carnicero en reses de novillos Criollos y cruzas $5 / 8$ Hereford-Nelore: peso y composición de músculo, grasa y hueso en cortes minoristas. Ganado Bovino Criollo. Tomo 2. pág 4962. Buenos Aires, Editorial Orientación gráfica. 
Garriz, C.A., Suárez, V., Gallinger, M.M., Bussetti, M., Carduza, F. y Rivera M.I. 1998a. Peso de faena y composición corporal en novillos puros y cruzas Criollo Argentino. Rev. Arg. Prod. Anim. 18 (Supl. 1): 261-262.

Garriz, C.A., Valy, F., Ludden, B., Parodi, J., Gallinger, M.M. y M. Marcelia. 1984. Producción de carne en zonas marginales (NOA) Evaluación del rendimiento carnicero en reses de novillos de raza Criolla Argentina y cruzas. Rev. Arg. Prod. Anim. 4 (5): 597-608.

Grajales, H., Hernández A. y E. Prieto. 2006. Edad y peso a la pubertad y su relación con la eficiencia reproductiva de grupos raciales bovinos en el trópico colombiano. Livestock Research for Rural Development 18 (10): 1-14.

Gree, R.C., Whitman, R.W., Staigmiller, R.B. y Anderson, D.C. 1983. Estimating the impact of management decisions on the occurrence of puberty in beef heifers. J. Anim. Sci. 56:30. https:/ / doi.org/ 10.2527/ jas1983.56130x

Holgado, F. D., Fernández, J. L., Ortega, M. F. 2017b. Influencia del peso de servicio a los 15 meses sobre el peso al nacer y ganancia predestete de las crías en Criollo argentino. AICA 10 (2017): 57-62.

Holgado, F. D., Fernández, J. L., Ortega, M. F. 2017c. Caracterización del crecimiento de vaquillonas Criollo argentino con servicio a los 15 meses de edad. AICA 10 (2017): 63-68.

Holgado, F.D. y A. E. Rabasa. 2001. Herencia del carácter "sin cuernos" en el bovino Criollo Argentino. Zootecnia Trop. 19(2): 185-190.

Holgado, F.D. y M. F. Ortega. 2013. Peso adulto del ganado bovino criollo argentino. XXIII Reunión de la ALPA. IV Congreso Internacional de Producción Animal Tropical. La Habana, Cuba, 2013. Pág 928930.

Holgado, F.D. y M.F. Ortega. 2019. Caracterización productiva del bovino Criollo Argentino: periodo 2006-2016. Ediciones INTA, Colección Investigación, Desarrollo e Innovación. Buenos Aires, Argentina. ISBN 978-987-521-987-8.

Holgado, F.D., Fernández, J.L., Ortega, M. F. 2017a. Efecto del peso de servicio a los 15 meses en la fertilidad de vaquillonas de la raza Criollo Argentino. AICA 10 (2017): 51-56.

Holgado, F.D., Martínez B., Díaz A., Soraire J. 2016b. Desgaste dentario en función de la edad en bovino Criollo Argentino. AICA 8 (2016):1-4.

Holgado, F.D., Martínez, B., Díaz, A. y Soraire J. 2016a. Influencia del fenotipo de la madre en el peso al nacimiento y ganancia de peso en terneros Criollo Argentino. AICA 8 (2016): 13-15.
Holgado, F.D., Martínez, R., Cantarella, G, Ortega M.F. y Fernández J.L. 2019. Influencia de la condición corporal al parto y al ingreso a servicio sobre el intervalo entre partos en vacas Criollas adultas. AICA 14 (2019): 60-63.

Holgado, F.D., Ortega M.F. 2015c. Caracterización de de toros adultos de la raza Criollo Argentino: peso corporal, alzada y circunferencia escrotal. AICA 6 (2015): 172-177.

Holgado, F.D., Ortega M.F. y Cantarella G. 2019. Efectos ambientales sobre los pesos al nacer y al destete en la raza bovina Criollo argentino. AICA 14 (2019): 64-68

Holgado, F.D., Ortega, M.F., Fernández J. 2015a. Caracterización cefálica y auricular de vacas Criollo Argentino con y sin cuernos. AICA 6 (2015): 329-333.

Holgado, F.D., Ortega, M.F., Fernández J. 2015b. Evolución con la edad de diferentes medidas corporales en hembras bovinas de la raza Criollo argentino. AICA 6 (2015): 178-183.

Holgado, F.D., Rabasa A.E., Ortega M.F. 2019. Longitud de prepucio en la raza bovina Criollo Argentino. AICA 14 (2019): 69-72.

Holgado, F.D., Rabasa de Sal Paz, A. y F. Sal Paz. 1988. Mortalidad predestete en distintos genotipos bovinos. Mendeliana 8(2): 123-134. ISSN 0325-223X.

Mariante, A.S. 1993. Conservación de bovinos Crioulo no Brasil. Diálogo XXXV, Evaluación y elección de biotipos de acuerdo a los sistemas de producción. IICA PROCISUR, Montevideo, Uruguay, pág 137142.

Mc Andrews, J.M., Stroud C.M., Mac Donald R.D., Hymer W.C. and Deaver D.R. 1993. Age related changes in the secretion of growth hormone in vivo and in vitro in infantile and prepubertal Holstein bull calves. Journal of endocrinology 139: 307-315. https:/ / doi.org/ 10.1677/ joe.0.1390307

Namur, P., C. Ferrando, E., Paloma, E. Orionte, y D. Leguiza. 2004. Recría de terneros Criollos y Aberdeen Angus en Buffel Grass. En Ganado Bovino Criollo, tomo 5, pág 7-12. Buenos Aires. Ed Orientación Gráfica.

Ortega, M.F., Holgado, F.D. 2017. Resistencia genética a a garrapata común del bovino en reproductores de raza Criollo Argentino. Actas del Congreso de Sociedad Argentina de Genética. Catamarca, Argentina.

Poli, M.A. 1993. Longitud y forma de las orejas en el bovino Criollo Argentino. Aspectos comparativos con Nelore. En Ganado Bovino Criollo, tomo 3, pág 79-90. Buenos Aires. Ed Orientación Gráfica.

Rabasa C., A. Rabasa de Sal Paz, F. Sal Paz, F. Bergmann y S.L. Rabasa. 1986. Genética de pelajes 
en bovinos Criollos. Ganado Bovino Criollo, tomo 1. pág 170-182. Buenos Aires. Ed Orientación Gráf.

Rabasa, A.E., Holgado, F., Poli M. A. 2005. Bovino Criollo de Argentina: Diferentes aspectos en sus avances en la caracterización. Agrociencia, IX, (2-3): 473-477.

Rabasa, S.L. 1986. Importancia relativa de los componentes de la producción. En Ganado Bovino Criollo, tomo 1, pág 151-159. Buenos Aires. Ed Orientación Gráfica.

Roy J.H.B. 1974. El ternero: nutrición y patología. II tomo. La Habana. Editorial Organismos 13-26 pp.

Sal Paz de, A. R., F.D. Holgado y S. L. 1995. Base genética de la resistencia al cáncer de ojo en bovinos. Mendeliana, 1995, 12 (1): 51-58.

Sal Paz, F.P. 1986. El ganado Criollo argentino: definición y principales características. Ganado Bovino Criollo, tomo 1, pág 3-7. Buenos Aires. Ed Orientación Gráf.
Torres Bruchamann, E. 1977. Atlas agroclimático y bioclimático de Tucumán (2a parte). Publicación Especial $\mathrm{N}^{\circ} 10$, Universidad Nacional de Tucumán, $13 p$.

Wilkins, J.V., Martínez, L. y Rojas, F. 1982. El ganado vacuno Criollo. CIAT. Santa Cruz, Bolivia, Documento № 33 .

Wilkins, J.V., y Rojas, F. 1993. Programa de mejoramiento genético bovino Criollo en Santa Cruz, Bolivia. Diálogo XXXV, Evaluación y elección de biotipos de acuerdo a los sistemas de producción. IICA PROCISUR, Montevideo, Uruguay, pág 129-133.

Zimerman, M. Bottegal, D., Oliverio, M.L., Ferrario, J. J. Ortega, M.F., Martínez, S., Royo, V., Molina, A., Holgado F., Fernandez Salom, M.J. Taboada, N. y Nasca J.A. 2019 b. Desempeño productivo y calidad de carne de novillos Braford, Criollo, Argentino, y F1 terminados a corral. Rev. Arg. Prod. Anim. 39 (Supl. 1): 81. 